\title{
Breaking the symmetry of a Brownian motor with symmetric potentials
}

\author{
H Hagman, M Zelan, C M Dion \\ Department of Physics, Umeå University, SE-901 87 Umeå, Sweden. \\ E-mail: claude.dion@tp.umu.se
}

\begin{abstract}
The directed transport of Brownian particles requires a system with an asymmetry and with non-equilibrium noise. We here investigate numerically alternative ways of fulfilling these requirements for a two-state Brownian motor, realised with Brownian particles alternating between two phaseshifted, symmetric potentials. We show that, besides the previously known spatiotemporal asymmetry based on unequal transfer rates between the potentials, inequalities in the potential depths, the frictions, or the equilibrium temperatures of the two potentials also generate the required asymmetry. We also show that the effects of the thermal noise and the noise of the transfer's randomness depend on the way the asymmetry is induced.
\end{abstract}

PACS numbers: 05.60.Cd, 05.40.Jc, 37.10.Jk

Submitted to: J. Phys. A: Math. Gen.

\section{Introduction}

Transport phenomena are ubiquitous in nature and are an interesting physical problem. As the thermal noise becomes increasingly important for the dynamics at shorter length scales, the control and the theoretical treatment of the transport generally becomes more complicated. Brownian motors do, however, take advantage of these random fluctuations, as they channel them into useful energy in the absence of bias forces 1, 2, 3, 4, 5. This makes them interesting from the point of view of statistical physics, and they have been proposed as the basis of a large number of transport phenomena in biological structures [1, 2, 6, 7, 8,

Brownian particles 9] in a periodic potential can be subjected to directed transport provided that two requirements are fulfilled: (i) the system has to possess an asymmetry, in accordance with the Curie principle [10], i.e., the trapping potentials have to present at least a spatial or spatio-temporal asymmetry; (ii) the system has to be out of thermal equilibrium, in agreement with the second law of thermodynamics [11.

In addition to the potentials, a friction force is usually present, the combination of the two localising the particles close to the potential minima, along with a diffusive force that is the source of the Brownian motion. This diffusive force is often treated by letting the particles interact with a heat bath of temperature $T$. The required asymmetry is usually included in the potential, e.g., as is the case for sawtooth 
potentials. To satisfy the second requirement stated above, the equilibrium between the particles and the heat bath has to be broken for drifts to be induced. This is usually done by non-adiabatically changing a parameter of the potential, e.g., the spatial phase in a rocked ratchet or the potential depth in a flashing ratchet, in a time-periodic fashion 11. The latter is equivalent to the case where the particles can be found in different states, each with its own characteristic static potential, and where it is the state that evolves dynamically.

As an alternative to an asymmetric potential, it is possible to achieved a biased drift for a multi-state particle using symmetrical potentials that are properly shifted between the states (see, e.g., 12, 13, 14, 15, 16]). Directed transport is then achieved through a combination of the shift of the potentials and inequalities in the rates of change of the states, resulting in a combined spatio-temporal asymmetry. To understand the processes involved, it can be convenient to consider this change of state as a transfer from one potential to another.

Such a Brownian motor, where the asymmetry is given by a combination of a non-zero relative spatial phase and unequal transfer rates between only two symmetric, periodic potentials, has been demonstrated both numerically [17] and experimentally [18, 19, 20, including with real-time control of the drift [21]. The original idea of using two phase-shifted symmetric potentials [17. (along with its implementation [18, 19, 20, 21]) was based on laser-cooled atoms interacting with two optical lattices, created from the interference of laser beams [22]. As such, it was assumed that the asymmetry coming from unequal transfer rates between the two phase-shifted optical lattices, as occurs "naturally" in the system, was the asymmetry driving the Brownian motor [17. However, this is far from the only asymmetry present in the system. As the frequency and irradiance of the lasers making each optical lattice can be adjusted independently, inequalities between the potentials depths, the diffusion, and the friction are usually present and can be modified by varying these parameters.

From a more general point of view, this two-state Brownian motor is a complex system, as the symmetry can be broken by any inequality between the two potentials, in combination with a relative spatial phase shift. That is, instead of unequal transfer rates, inequalities in the equilibrium temperature, the potential depth, or the friction could be used. These multiple ways of breaking the symmetry have not yet been investigated. Moreover, in the original system, the transfers (or changes of state) are random, as they originate from spontaneous emission. This adds a source of randomness to the system, in addition to the Brownian motion of the particles. We thus investigate below the role of these two sources of fluctuations and of the different kinds of asymmetries using numerical simulations.

\section{Model}

The system considered consists of a Brownian particle interacting with either of two symmetric potentials,

$$
\begin{aligned}
U_{1} & =\frac{A_{1}}{2}[1-\cos (k x)], \\
U_{2} & =\frac{A_{2}}{2}[1-\cos (k x+\varphi)],
\end{aligned}
$$

where $A_{i}$ are the potential depths, $k$ is the wave number and $\varphi$ the relative spatial phase of the potentials. We hereafter scale length such that $k=2 \pi$. The particle will 


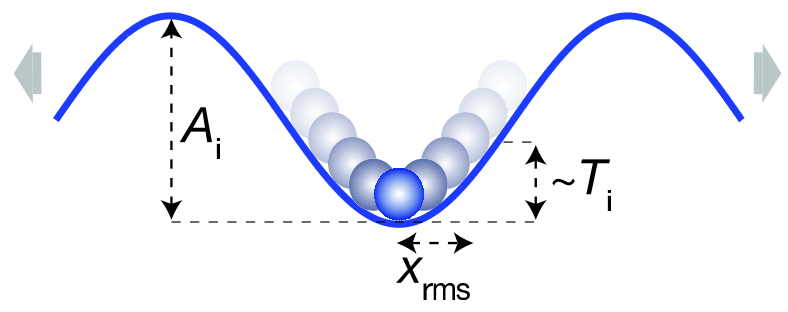

Figure 1. Schematic illustration of the distribution of a Brownian particle in a periodic potential with friction and diffusive forces present. At equilibrium, the distribution will be centred around potential minima with a velocity spread proportional to the equilibrium temperature $T$, and a position spread, $x_{\mathrm{rms}}$, dependent on the temperature $T$ and potential depth $A$. Even though the thermal energy is less than the potential depth, the spread of the distribution will lead to a diffusion in the potential, indicated with thick horizontal arrows in the figure.

be transferred between the states (the potentials) with transfer rates $\Gamma_{1 \rightarrow 2}=1 / \tau_{1}$ and $\Gamma_{2 \rightarrow 1}=1 / \tau_{2}$, where $\tau_{i}$ are the lifetimes of the potentials, and is submitted to state-dependent friction, $\alpha_{i} \dot{x}$, and diffusive, $\xi_{i}(t)$, forces. This can be summed up in the Langevin equation of motion 23

$$
\ddot{x}=-\nabla_{x} U_{i}(x)-\alpha_{i} \dot{x}+\xi_{i}(t),
$$

where $\xi_{i}(t)$ is a Gaussian white noise satisfying the relations $\left\langle\xi_{i}(t)\right\rangle=0$ and $\left\langle\xi_{i}(t) \xi_{i}\left(t^{\prime}\right)\right\rangle=2 \alpha_{i} T_{i} \delta\left(t-t^{\prime}\right)$, using a system of units where the mass of the Brownian particle $m=1$ and Boltzmann's constant $k_{\mathrm{B}}=1$. The index $i \in\{1,2\}$ is either a random variable or changes at fixed times, with a rate of change set by the transfer rates mentioned above. When it is random, we consider the change of state as being a source of noise, as it leads to non-deterministic, noisy dynamics, in addition to $\xi_{i}(t)$ which we term thermal noise.

The numerical integration of (22) is done with the BBK method 24. We use time steps of size 0.01, random initial positions and velocities, and run the simulations for 1000 or 2000 particles for about 40000 time steps.

The relative magnitude of the parameters are chosen to achieve a Brownian motor and, more specifically, to mimic the experimental system used in [18, 19, 20, 21. This means that the diffusive force should be large enough to clearly influence the dynamics, while small enough for the trapping potential to be relevant. As a default setting, we take $T_{1}=T_{2}=1, A_{1}=A_{2}=4$, and $\varphi=2 \pi / 3$ (the choice of the latter is explained in section 3). This gives a potential depth that is four times larger than the thermal energy, $A_{i} / T_{i}=4$, such that the particles are generally localised close to the potential minima but occasionally diffuse to neighbouring wells. A schematic illustration of the distribution of a Brownian particle in these conditions is shown in figure 1, with the width of the distribution characterised by the root-mean-square position (or standard deviation) $x_{\mathrm{rms}} \equiv \sqrt{\left\langle x^{2}\right\rangle-\langle x\rangle^{2}}$ (the position is to be understood as taken modulo $2 \pi / k$, with \langle\rangle denoting an ensemble average).

The simulations are done for moderate friction, with default values of $\alpha_{1}=$ $\alpha_{2}=1 / 2$, such that the system is not overdamped, in contrast to most other investigations of Brownian motor systems. The equilibrium properties are similar for both underdamped and overdamped systems, but the route to equilibrium differs significantly. This can be illustrated by considering a moderately damped particle starting at the slope of the potential. With time, the position and velocity spread 

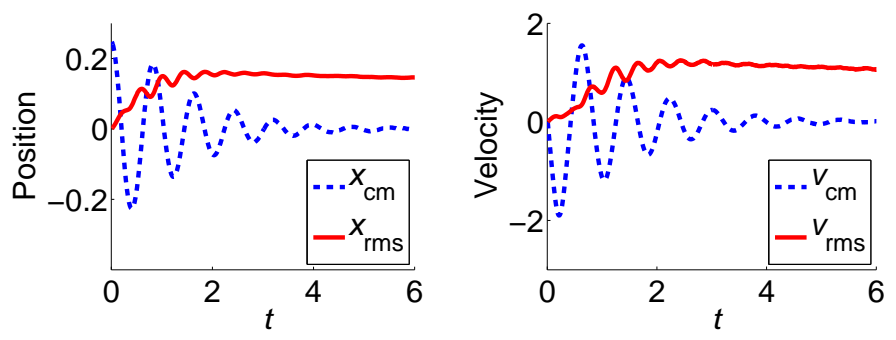

Figure 2. Time evolution of the position and velocity distribution of a Brownian particle starting on a slope of a periodic potential with a finite friction and diffusive force. The initial oscillations of $x_{\mathrm{cm}}$ and $v_{\mathrm{cm}}$ are damped on the same time scale as the widths of the distributions, $x_{\mathrm{rms}}$ and $v_{\mathrm{rms}}$, reach their equilibrium values.

will increase toward the equilibrium values, see figure 2. The average position, $x_{\mathrm{cm}} \equiv\langle x \quad(\bmod 2 \pi / k)\rangle$, and velocity $v_{\mathrm{cm}} \equiv\langle v\rangle$ will, on the same time scale, perform a damped oscillation around the equilibrium value of zero $(x=0$ being the centre of the well), see figure 2 In this case, the particle could be said to gradually lose memory of earlier positions or velocities. For an overdamped system, no such oscillations take place, as any memory of earlier positions or velocities are quickly lost.

The relation between the inter-potential transfer rates and the time to reach equilibrium crucially influences the distribution of positions at the instant of the transfer between the potentials. To study the effects of the oscillations before equilibrium is reached (with friction $\alpha=1 / 2$ ), transfer rates $\Gamma_{i \rightarrow j}>1 / 4$ should be used, while for studies under equilibrium conditions $\Gamma_{i \rightarrow j} \leq 1 / 4$ be used, see figure 2 The inter-potential transfer can be done in two ways: either at fixed times, analogous to the flashing ratchet model, or with random transfer times, adding another source of noise to the Brownian system. The difference in the resulting dynamics will be investigated and discussed later in this paper.

When the transfer times are relatively short compared to the time required to reach equilibrium in a given state (figure 2), the actual kinetic temperature of the particles in state $i$ will be greater than the equilibrium temperature $T_{i}$. Therefore, when we discuss the equilibrium properties in this case, we mean the equilibrium that would be reached in absence of the fast transfers, not the steady-state properties of the system.

\section{Directed transport with asymmetric transfer rates}

A necessary condition for directed transport is for the two potentials to be out of phase (i.e., $\varphi \neq n \pi$, with $n$ an integer), but this is not sufficient to completely break the symmetry of the system. Indeed, it was shown that no drift can be induced if such a system is overdamped [13. Otherwise, unequal transfer rates $\left(\Gamma_{1 \rightarrow 2} \neq \Gamma_{2 \rightarrow 1}\right)$ will result in directed transport, as shown with both simulations [17] and experiments $[18,19,20,21$. In both these cases, the times at which the particle changes state are random. We show in figure 3 the dependence of the average transport velocity on the relative spatial phase for the model described above with $\Gamma_{1 \rightarrow 2}=10 \Gamma_{2 \rightarrow 1}=1$. We point out that it is this case (unequal transfer rates and random transfer times) that was assumed to most closely resemble the experimental implementation with cold caesium atoms [18, 19, 20, 21], and was thus the only case 


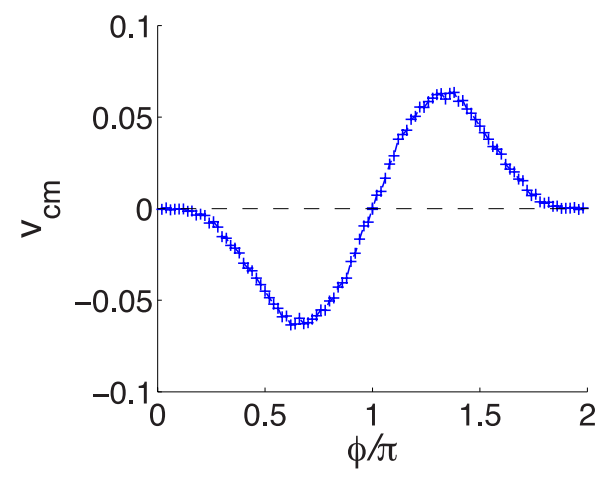

Figure 3. Effect of the relative spatial phase of the two potentials on the average drift velocity of the particles. The potentials are identical, but with unequal transfer rates between them, $\Gamma_{1 \rightarrow 2}=10 \Gamma_{2 \rightarrow 1}=1$.

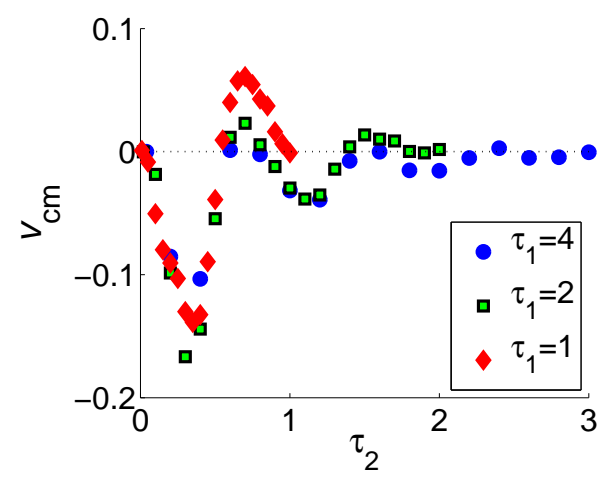

Figure 4. Directed transport for fixed asymmetric transfer times, plotted as the centre-of-mass velocity $v_{\mathrm{cm}}$ vs the lifetime in potential $2, \tau_{2}$, for three different values of the lifetime in potential 1: $\tau_{1}=1$ (diamonds), $\tau_{1}=2$ (squares), and $\tau_{1}=4$ (circles).

studied theoretically in previous work [17, 19, 20, 25].

To better understand the dynamics of the induced drifts, we set the relative spatial phase to the value that optimises the drift in the previous case, $\varphi=2 \pi / 3$ (figure 3), and now consider the effect of a varying asymmetry in the transfer rates. This is done below for both fixed and random transfer times.

\subsection{Fixed transfer times}

By letting the transfers occur at fixed times, a single source of randomness remains, that due to $T>0$, and the system is somewhat simplified. The asymmetry is here scanned by varying $\tau_{2}$ from about $0.01 \tau_{1}$ (highly asymmetric) to $\tau_{1}$ (symmetric), for three different values of $\tau_{1}$. The resulting average velocity of the induced drifts can be seen in figure 4 Since the two states are identical, apart from the transfer rates, they have equal equilibrium properties. The drifts are hence a direct consequence of the underdamped oscillations seen in figure 2 These can induce drifts in two related ways, as illustrated in figure 5. Consider a particle located close to the bottom of a 


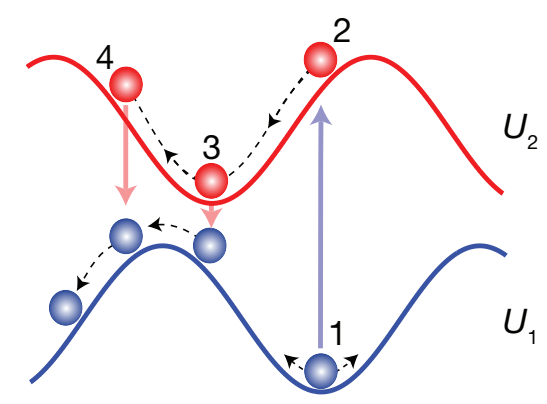

Figure 5. Schematic illustration of the directed transport mechanism for unequal transfer rates. (1) The particle has spent a long enough time in potential 1 to be close to equilibrium and be well localised close to the bottom of a well. (2) The particle gets transferred to a slope in potential 2, and starts moving. (3) The particle has been accelerated to the bottom of a well in potential 2 and gained momentum. If the particle is transferred back to potential 1, this momentum may be enough to overcome the barrier to the next well. (4) If the particle moves to the other slope of the well in potential 2 before being transferred back to potential 1 , the barrier to the next well in potential 1 has now been passed.

well of potential 1. As it is transferred to potential 2, it will end up on a slope, and start moving towards the minimum. If the particle moves past the minimum and up on the other slope before being transferred back, it can go beyond the point of the potential barrier of potential 1. Even if it comes back to the same well in potential 1, the momentum gained can be enough to overcome the barrier in potential 1.

In figure 4 a clear oscillatory pattern in the drift velocity's dependence on $\tau_{2}$ is seen. The oscillations have the same periodicity as in figure 2, and their amplitude follows the same decaying envelope. For $\tau_{1}=4$, four peaks in the drift velocity can be seen, all in the same direction. For smaller $\tau_{1}$, velocity peaks in the other direction can be seen, since the pre-equilibrium oscillations are now large enough to matter in potential 1, and the average drift hence gets a complex dependence on the oscillations in both potentials.

These drifts are a consequence of the finite damping and this scheme would hence not work in an overdamped case [13. Nevertheless, clear similarities with the overdamped flashing and rocked ratchets can be seen, where large increases in the diffusion and the drift have been reported for certain resonances in the flashing rates 26, 27, 28. For an asymmetry built on unequal transfer rates with deterministic transfer times, this works by matching transfer times and oscillation frequencies. In the deterministic limit, as the position spread of the particles is small, unidirectional drifts can thus be achieved. This situation is similar to a conventional internal combustion engine, where the timing of the ignition with the position of the piston is crucial, and as such the system does not correspond per se to a Brownian motor.

\subsection{Random transfer times}

Here the inter-potential transfers occur at random times, adding a source of randomness to the dynamics. With these extra fluctuations present, the average drift velocity is monitored for a varying average lifetime in potential $2, \tau_{2}$, for three different average lifetimes in potential $1, \tau_{1}$. The results are seen in figure 6. To distinguish the effects of the two noise sources, the simulations are done for both $T=0$ and $T=1$. 
(a)

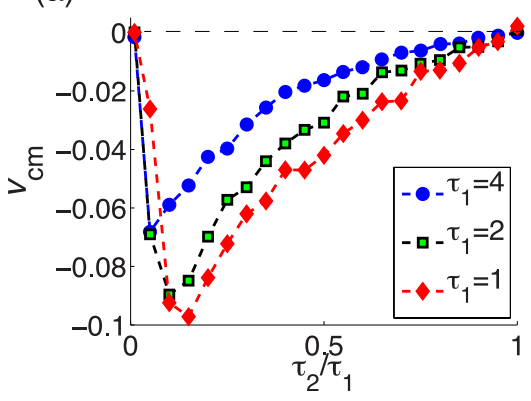

(b)

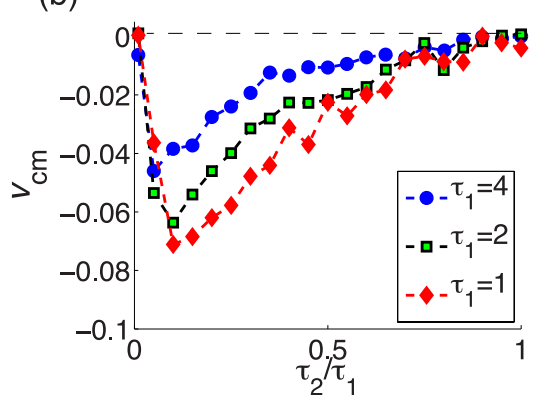

Figure 6. Directed transport for random asymmetric transfer times, plotted as the centre-of-mass velocity $v_{c m}$ vs the lifetime in potential $2, \tau_{2}$, for three different values of the lifetime in potential 1: $\tau_{1}=1$ (diamonds), $\tau_{1}=2$ (squares), and $\tau_{1}=4$ (circles). (a) $T=0$, showing drifts for all $\tau_{1} \neq \tau_{2}$. (b) $T=1$, resulting in the same structure as for $T=0$, but with slightly smaller drifts. The lines serve as a guide to the eye.

All resonance behaviour seen in figure 4 has disappeared, and all drifts are induced in the same direction, even for short transfer times. That is, the drifts are induced due to asymmetries in position or velocity space rather than by transfers at deterministic positions. In other words, since the transfer times are now a distribution rather than specific times, there is an uneven competition between processes that lead to diffusion in both directions. The similarity to conventional engines is lost and the behaviour is that of a "true" Brownian motor. Note that similar results are achieved both with and without the thermal noise, and that the equilibrium noise slows down the drifts rather than enhance them. However, random transfer times eliminate the need for a precise timing of the transfers.

Since it is the noise from the random transfers that is rectified, rather than the thermal noise, drifts can be induced for particles that are initially at rest. This is in clear contrast to the flashing ratchet, where the drift comes from an enhancement of the thermal noise. For a flashing ratchet, zero velocity corresponds to an absorbing state for $T=0$, even if random flashing would be used.

Since the equilibrium states are equal, the drifts are a consequence of the finite damping, as was also the case for fixed transfer times, and no drifts would be induced if the motion became overdamped [13. We will now explore the possibility of inducing drifts with equal transfer rates but unequal equilibrium properties in the two states.

\section{Breaking the symmetry of time-symmetric potentials}

We now consider induced drifts with equal transfer rates, $\Gamma_{1 \rightarrow 2}=\Gamma_{2 \rightarrow 1}$, by introducing inequalities in the equilibrium states. The equilibrium states have zero average velocity and an average position centred in the wells, see figures 10 and 2 . Therefore, no resonances in the timing of the transfer can be found after the equilibrium has been reached. The equilibrium states are characterised by the widths of the velocity and position distributions in each well. In our model, these depend on the temperatures and the potential depths of the two states. We now investigate the effects of asymmetries in these parameters. 


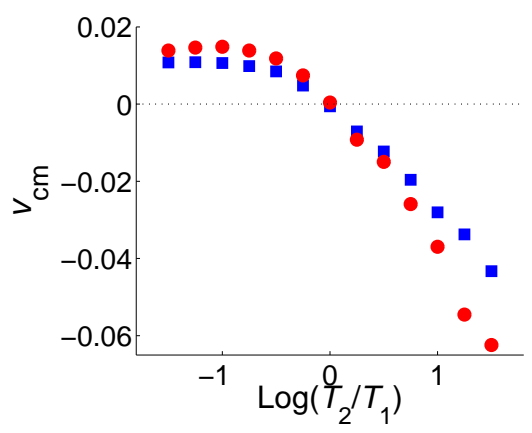

Figure 7. Drift velocity resulting from unequal temperatures in the two potentials, for random (squares) and fixed (circles) transfer times $\left(\tau_{1}=\tau_{2}=10\right)$. $T_{1}=1$ and $T_{2}$ is varied.

\subsection{States with different temperatures}

The temperature determines the width of the equilibrium distribution of both the velocity and position within a well. In potentials of equal depth, different temperatures thus result in differences between the distributions in the two potentials. This, together with a relative spatial phase, breaks the symmetry and can hence induce drifts.

To demonstrate this, $T_{2}$ is varied for fixed $T_{1}=1$, in otherwise identical potentials. Since it is the equilibrium properties that are interesting, transfer times long enough to reach equilibrium are used, $\tau_{1}=\tau_{2}=10$. The results for both deterministic and random transfer times are seen in figure 7 . Slightly higher drift velocities for deterministic transfer times are obtained. This is expected since all transfers occur when the two unequal equilibrium states have been reached for the case of deterministic transfers, while for random times a few transfers occur before equilibrium has been reached, which is on average unfavourable.

The symmetry breaking can here be illustrated by considering the equilibrium distributions in the two potentials, see figure 8 Just before a transfer, the position distribution will be centred around a minimum in potential 1 with a certain spread. If the relative spatial phase is non-zero and less than $\pi$, the position of a minimum in potential 1 is located in between a minimum and a maximum of potential 2, see figure 8. If the spread is sufficiently small, the particle will, with a probability close to unity, end up after the transfer on the slope pointing toward the closest minimum in potential 2. However, if the spread is large enough, there is a finite probability to end up on the slope of the neighbouring minimum, leading to a diffusion of the particles. If the distributions in the two potentials have unequal spreads, the increased diffusion will be biased in one direction. To further investigate the mechanism of this biased diffusion, we define the difference between the probability of ending up in a neighbouring minimum, $P_{i \rightarrow j}^{\text {neighbour }}$, for the two transitions as

$$
\Delta \equiv P_{1 \rightarrow 2}^{\text {neighbour }}-P_{2 \rightarrow 1}^{\text {neighbour }} .
$$

The value of $\Delta$ can be extracted from the simulations by keeping track of the positions at which the transfers occur. If $\Delta$ is plotted for the same asymmetries as in figure 7 the same basic dependency is recreated, see figure 9. A small deviation is visible for large temperatures. This could either be due the states having different velocity 


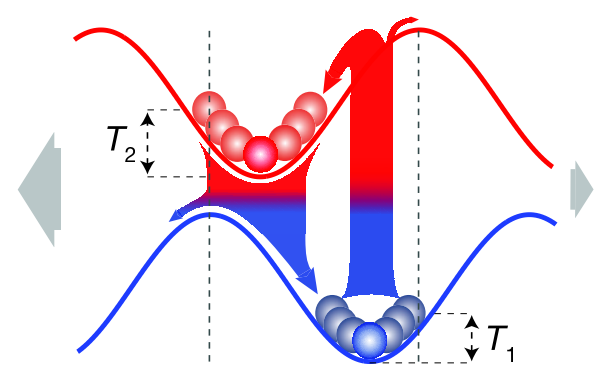

Figure 8. Schematic representation of different temperatures in the two potentials. The spread of the position distributions together with the relative spatial phase will lead to an increased diffusion of the particles due to the transfers. The unequal widths of the distributions bias this diffusion in one direction.

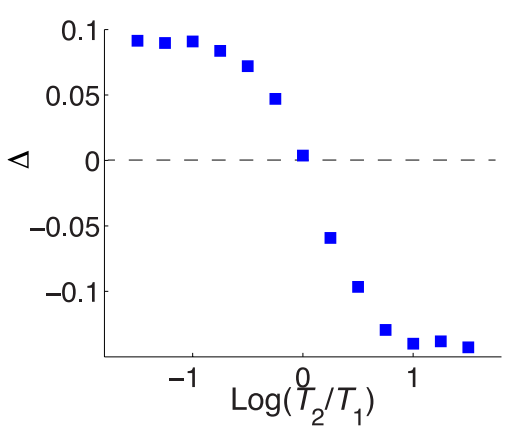

Figure 9. Asymmetry in the probability of a particle ending up in a neighbouring well, $\Delta$ (3), resulting from unequal temperatures in the two potentials $\left(T_{1}=1\right.$ and $T_{2}$ is varied).

distributions or, since the finite damping allows for multi-well flights, a difference in the average flight length in each direction.

In contrast to the case of unequal transfer rates, it is here the thermal noise, which creates the spread of the equilibrium distributions, that is enhanced and rectified, just as for a flashing ratchet. This type of symmetry breaking would hence also work for an overdamped system. Drifts from two heat baths with different temperatures are analogous to the Feynman-Smoluchowski thought experiment [11.

\subsection{States with different potential depths}

The symmetry can be broken by unequal potential depths as well. While this is formally equivalent to having different temperatures corresponding to each state, as both cases correspond to unequal energy scales for the two states, treating it independently allows us to make clearer the role of the thermal noise.

As with temperature, the potential depth affects the equilibrium distribution in position: a larger depth gives a narrower spread in position. In figure 10, the drift velocity is shown for a varying depth of potential 2. This is done for random transfer times, and for both for $T=0$ and $T=1$. To allow the system to reach equilibrium between transfers, the transfer times are again set to $\tau_{1}=\tau_{2}=10$. Note that the dependence of the drift on $A_{2} / A_{1}$ is inverted for the two temperatures, which can be 


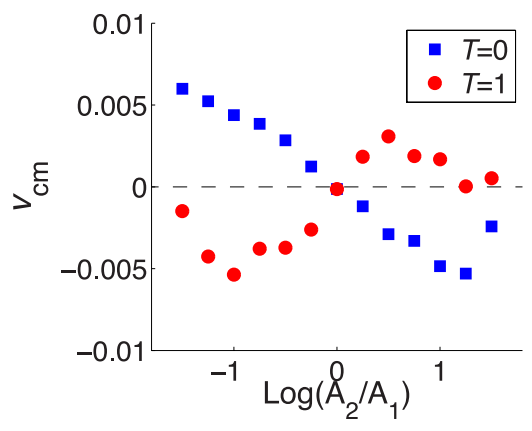

Figure 10. Drift velocity resulting from unequal potential depths $\left(A_{1}=4\right.$ and $A_{2}$ is varied), for $T=0$ (squares) and $T=1$ (circles) with random transfer times $\Gamma_{1 \rightarrow 2}=\Gamma_{2 \rightarrow 1}=1 / 10$. The equilibrium noise $(T)$ inverses the average drift velocity's dependence on $A_{2} / A_{1}$.

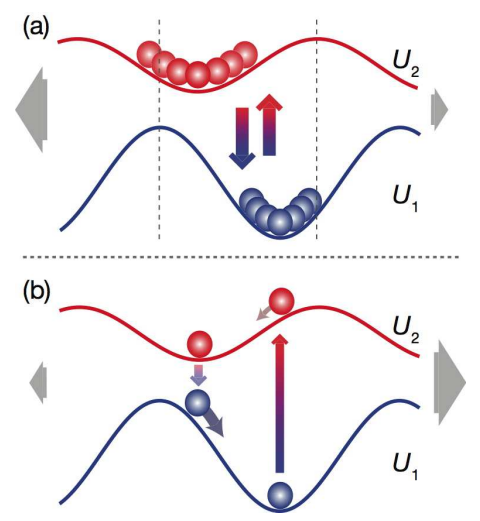

Figure 11. Schematic representation of potentials with different depths. (a) The spread of the position in the equilibrium distributions together with the relative spatial phase will lead to an increased diffusion of the particles. Due to the unequal widths of the distributions, this diffusion will be biased in one direction for long transfer times. (b) The difference in potential depths gives different slopes and different velocities for short transfer times. The two effects and (a) and (b) have opposite directions.

explained as follows. For finite but equal temperatures, the position spread will be greater for the shallower potential and, for the current set of parameters, this leads to a drift in the negative direction when $A_{2}<A_{1}$ (see figure 11(a)) and vice versa. When $T=0$, if the transfer times are short enough, the dynamics are dominated by the velocity acquired when changing potential, which is greater when going to the deeper potential, as illustrated in figure 11(b), resulting a drift in the positive direction for $A_{2}<A_{1}$. For longer transfer times, where the system would have to reach equilibrium between transfers, no drift would be observed for $T=0$. This phenomenon of purely noise-induced reversal of the drift has also been reported for rocked ratchets [26].

To further investigate the noise-induced reversal of the drift, the temperatures are scanned from 0.01 to unity, for an asymmetry $A_{2} / A_{1}=1 / 10$ (resulting large drifts in both directions, see figure 10). The drift achieved is shown in figure 12, together with $\Delta$ (3), where a correlation between the two is evident. 


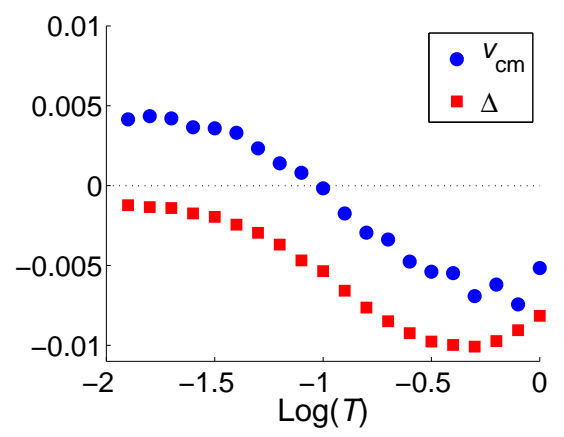

Figure 12. Drift velocity $v_{\mathrm{cm}}$ and probability asymmetry $\Delta$ (3) for different temperatures $\left(T_{1}=T_{2}=T\right)$, for $A_{2} / A_{1}=1 / 10$.

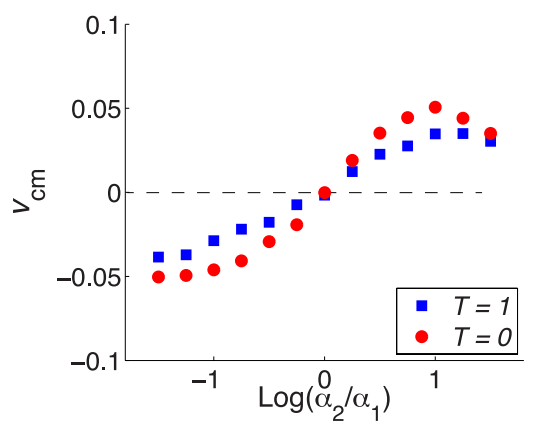

Figure 13. Drift velocity for different friction coefficients in the two potentials for $T=0$ (circles) and $T=1$ (squares). $\alpha_{2}$ is varied for constant $\alpha_{1}=5$.

With fixed transfer times, the dominant effect, and thus the direction of the drift, depends on the value of the transfer rate. This is only possible for the underdamped case, where resonances due to transient effects can be found, as for an overdamped system only the equilibrium properties are of importance.

\subsection{States with different friction}

In the Langevin formulation of the problem (2), friction does not change the temperature of the equilibrium states (indeed, friction can be removed from the equations by the rescaling $t \rightarrow \alpha t$ ). What the friction does change is the decay time of the pre-equilibrium oscillations (figure 2). Unequal frictions can hence induce drifts, and in figure 13 drifts in both directions are evident for a varying $\alpha_{2} / \alpha_{1}$. Since the drifts do not depend here on unequal equilibrium states, this scheme works for both $T=0$ and $T=1$.

This asymmetry is closely related to the case of unequal transfer rates. This is clearly seen by using fixed equal transfer times, $\tau_{1}=\tau_{2}=\tau$, and scanning these for a friction inequality $\alpha_{1}=5, \alpha_{2}=1 / 2$, which gives large drifts, see figure 14. 


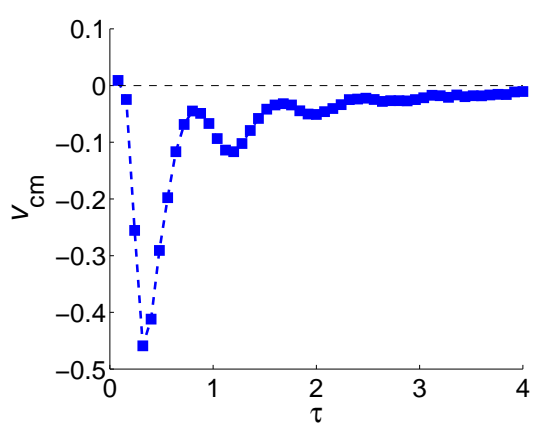

Figure 14. Drift velocity $v_{c m} v s$ the deterministic transfer time $\tau$ of both potentials for a friction inequality of $\alpha_{1}=5$ and $\alpha_{2}=1 / 2$. The line serves as a guide to the eye.

\section{Conclusion}

We have shown that two-state Brownian motors, realised with Brownian particles in symmetric potentials, are very rich systems, where the necessary spatio-temporal asymmetry required for a directed motion can be induced by a differentiation of either the two potential depths, the two temperatures, the two frictions, or the two lifetimes of the system. For the case of different transfer rates or different frictions, the random fluctuations associated with the random inter-potential transfer are shown to be the energy source, i.e., the noise that is rectified. For the case of different potential depths or different equilibrium temperatures, it is the thermal noise in each potential that was shown to be the relevant energy source. For the case of an asymmetry in the potential depth, a pure noise-induced reversal of the drift is also demonstrated.

The richness of this Brownian motor makes it flexible and clear similarities to several different types of systems can be seen, depending on the source of the asymmetry. It serves as a good exemplification of the statement of Pierre Curie that "when certain causes produce certain effects, the elements of symmetry of the causes must be found in the effects produced" [10, as in our system any asymmetry will lead to a directed motion. The results demonstrate also that the source of the symmetry breaking of a Brownian motor can be diverse, and indeed even many asymmetries can be present simultaneously, which would typically be the case for atoms in a double optical lattice [18, 19, 20, 21]. The increased understanding of the model as such also increases the understanding, and possibilities to optimise, the above-mentioned existing experimental realisation of the system.

\section{Acknowledgments}

C.M.D. gratefully acknowledges funding from the Swedish Research Council and Umeå University.

\section{References}

[1] Hänggi P and Marchesoni F 2009 Rev. Mod. Phys. 81387

[2] Astumian R D 1997 Science 276917

[3] Hänggi P, Marchesoni F and Nori F 2005 Ann. Phys. (Leipzig) 1451 
[4] Reimann P 2002 Phys. Rep. 36157

[5] Astumian R D and Hänggi P 2002 Phys. Today 55 (11) 33

[6] Linke H (ed) 2002 Ratchets and Brownian Motors: Basics, Experiments and Applications, Appl. Phys. A 75 (2)

[7] Jülicher F, Ajdari A and Prost J 1997 Rev. Mod. Phys. 691269

[8] Mogilner A and Oster G 2003 Biophys. J. 841591

[9] Einstein A 1906 Ann. Phys. 19371

[10] Curie P 1894 J. Phys. III (Paris) 3393

[11] Feynman R P, Lieghton R B and Sands M 1963 The Feynman Lectures on Physics vol. 1 (Reading, MA: Addison-Wesley)

[12] Chen Y D 1997 Phys. Rev. Lett. 793117

[13] Kanada R and Sasaki K 1999 J. Phys. Soc. Japan 683759

[14] Lee S H, Ladavac K, Polin M and Grier D G 2005 Phys. Rev. Lett. 94110601

[15] Lee S H and Grier D G 2005 Phys. Rev. E 71060102

[16] Gommers R, Bergamini S and Renzoni F 2005 Phys. Rev. Lett. 95073003

[17] Sanchez-Palencia L 2004 Phys. Rev. E 70011102

[18] Sjölund P, Petra S J H, Dion C M, Jonsell S, Nylén M, Sanchez-Palencia L and Kastberg A 2006 Phys. Rev. Lett. 96190602

[19] Sjölund P, Petra S J H, Dion C M, Jonsell S, Hagman H and Kastberg A 2007 Eur. Phys. J. D 44381

[20] Hagman H, Dion C M, Sjölund P, Petra S J H and Kastberg A 2008 EPL 8133001

[21] Hagman H, Zelan M, Dion C M and Kastberg A 2011 Phys. Rev. E 83 020101(R)

[22] Grynberg G and Robilliard C 2001 Phys. Rep. 355335

[23] Risken H 1989 The Fokker-Planck Equation 2nd edn (Berlin: Springer)

[24] Wang W and Skeel R D 2003 Mol. Phys. 1012149

[25] Dion C M, Sjölund P, Petra S J H, Jonsell S, Nylén M, Sanchez-Palencia L and Kastberg A 2008 Eur. Phys. J. Special Topics 14911

[26] Schreier M, Reimann P, Hänggi P and Pollak E 1998 Europhys. Lett. 44416

[27] Mu W, Liu Z, Luan L, Wang G, Spalding G C and Ketterson J B 2009 New J. Phys. 11103017

[28] Lin-Ru N, Yu-Lan G and Dong-Cheng M 2009 Chin. Phys. Lett. 26100505 